Original Article

\title{
Prediction of Infectious Complications after Percutaneous Nephrolithotomy
}

\author{
Raj Kumar Chhetri, Suman Baral, Neeraj Thapa \\ Department of Surgery, Lumbini Medical College, Palpa, Nepal \\ Correspondance: Dr Raj Kumar Chhetri, Associate Professor, Department of Surgery, Lumbini Medical College, Palpa, Nepal
}

Email: chhetrirkcdr@gmail.com

\begin{abstract}
Introduction: Post-operative infection is one of the most common and potentially life-threatening complications following percutaneous nephrolithotomy, ranging from Systemic inflammatory response syndrome (SIRS) to severe sepsis. It is reported to be the most common peri-operative cause of death. Despite taking utmost precautions, we come across major complications such as haemorrhage and urosepsis after percutaneous nephrolithotomy. This study aims to find the risk factors for infectious complications after percutaneous nephrolithotomy.
\end{abstract}

Methods: This was an observational, cross-sectional, analytical study carried out in the Department of Surgery of Lumbini Medical College and Teaching Hospital over a period of six months. Preoperative and intra-operative parameters of ninetyseven patients who underwent percutaneous nephrolithotomy were analysed to see the association between perioperative and intraoperative factors in development of post-operative infectious complications.

Results: Post-operatively, SIRS was observed in $28.9 \%$ of the patients and $6.2 \%$ developed post operative sepsis. Stone burden, types of stone, abnormal urinalysis and mean operative time were associated with post-operative SIRS while stone burden, abnormal urinalysis, positive urine culture and operation time were statistically associated with post-operative sepsis. In univariate analysis only mean operation time and mean stone burden were statistically associated in development of post operative SIRS and sepsis.

Conclusion: In the present study mean operation time and mean stone burden were found to be the predictive factors for post-operative infectious complications after percutaneous nephrolithotomy.

Key words: Operation time; Percutaneous nephrolithotomy; Systemic inflammatory response syndrome; Sepsis; Stone burden.

\section{Introduction}

Urinary stone disease is one of the most common benign urologic diseases affecting around $0.13 \%$ of the population with a lifetime incidence of $10 \% 1 .{ }^{1}$ Since its introduction by Fernström and Johansson for extracting renal calculi in 1976, percutaneous nephrolithotomy (PCNL) has become the gold standard of care for renal stones $^{2}$ and is one of the most common surgical procedures in urologic practice. ${ }^{3}$
While PCNL is regarded as safe procedure with less trauma and quicker recovery than the open nephrolithotomy, it is not without risk for significant complications with Clavien >II complications occurring in around $9 \%$ of cases including post-operative fever $(10.8 \%)$, bleeding $(7 \%)$, thoracic complication $(1.5 \%)$, sepsis $(0.5 \%)$, embolization $(0.4 \%)$, organ injury $(0.4 \%)$ and urinoma $(0.2 \%)^{1,4-6}$ Among the complications, postoperative infection is one of the most dreaded complications ranging from fever to severe 
sepsis. ${ }^{5,7}$ Urosepsis is a well-known and potentially lifethreatening complications following PCNL for renal calculi. ${ }^{7-8}$ which is reported to be the most common perioperative cause of death. ${ }^{2}$

PCNL is a routine operation done in the Department of Surgery of Lumbini Medical College and Teaching Hospital (LMCTH) for renal stones. Despite taking utmost precautions, we come across major complications such as haemorrhage and urosepsis beside minor complications after PCNL. So it is intended to carry out this study to find the risk factors for infective complications after PCNL.

\section{Methods}

This is an observational, cross-sectional, analytical study done in the Department of Surgery of LMCTH over a period of six months (February 2018 to July 2018). Study protocol was approved by the Institutional Review Committee (IRC-LMC 30-E/018). A special proforma was designed and filled up by surgical residents. A total of ninety-seven patients who were fit to undergo PCNL under general anaesthesia were included in the study. They were investigated with ultrasonography and intravenous urography to know the size and number of stones and their location in the kidney or upper ureter. Urinalysis and urine culture were routinely sent to detect the urinary tract infection preoperatively and pus cells more than 5/ HPF was considered as abnormal urinalysis. ${ }^{9}$ If the urine showed pus cells or are culture positive, they were given seven days course of antibiotics and re-evaluated. Once the urine is found to be culture negative then they were posted for the surgery. Patients were then admitted in the Surgery Department a day before surgery and intravenous antibiotics started.

Prophylactic third generation cephalosporin (cefoperazone) antibiotic were given according to the local guidelines of the hospital or on the basis of preoperative urine culture report one hour prior to induction of anaesthesia. After placing the patient in the lithotomy position, retrograde ureteric catheterisation with a 6-F open-ended ureteric catheter was performed under fluoroscopy guidance using a rigid cystoscope. All other parts of procedures were completed in the prone position. The selected calyx was accessed using C-arm fluoroscopy. The 8-G coaxial needle was placed in the preferred calyx. The floppy tipped guidewire was then passed into the collecting system through the needle. A working channel was established using a serial plastic dilator under fluoroscopy control upto 20F. Then the Amplatz sheath was placed over the dilated tract. A 20F nephroscope was then placed directly into the kidney through the Amplatz. The stones were fragmented using a pneumatic lithotripter. Forceps was used to remove stone fragments. The number and types of accesses depended on the size of the treated stones (staghorn stone vs single stone) and location (upper or lower pole). At the end of the procedure ureteric catheter was removed and a double J stent placed in the ureter. A 20F nephrostomy tube was placed through the Amplatz sheath and fixed to the skin after removal of the Amplatz sheath in all patients and clamped for $8 \mathrm{~h}$. Post operatively, all the patients were subjected for investigations like complete blood count, routine urinalysis and urine culture. Third generation cephalosporin (cefoperazone) was given according to the local guidelines of the hospital or on the basis of preoperative urine culture report. Nephrostomy tube was removed in $2^{\text {nd }}$ day, Foley's catheter on $3^{\text {rd }}$ day and the patient discharged if no features of SIRS or sepsis was observed.

Postoperatively, infectious complications including systemic inflammatory response syndrome (SIRS) with two or more of the following criteria such as temperature $>38^{\circ} \mathrm{C}$ or $\angle 36^{\circ} \mathrm{C}$, pulse rate $>90 / \mathrm{bpm}$, respiratory rate $>20 \mathrm{breaths} / \mathrm{min}$, white blood cell count $<4000 / \mathrm{mm}^{3}$ or $>12000 / \mathrm{mm}^{3}$; sepsis defined as SIRS with positive urine or blood culture were recorded. ${ }^{5,8}$ All the infectionrelated complications were recorded upto three weeks when the double $\mathrm{J}$ stent was removed.

SPSS version 16 was used to analyze the data. Chi square test, Fishers exact test, Student t test and univariate logistic regression analysis were applied with $\mathrm{P}$ value less than 0.05 being considered significant.

\section{Results}

There were 97 patients in the study which included 55 males and 42 females with the mean age of $34.39 \pm 11.84$ years. Mean age of the patients who developed postoperative SIRS was $30.89 \pm 7.714$ years and those who did not develop SIRS were $35.87 \pm 12.920$ years ( $P$ value 0.06 ). Among the males 37 developed SIRS while 32 female patients developed SIRS (P value 0.34).

Age of the patients who developed postoperative sepsis was $27.20 \pm 5.22$ years and those who did not develop sepsis were $34.78 \pm 12.00$ years ( $\mathrm{P}$ value 0.33 ). Five male and one female patient developed postoperative sepsis ( $\mathrm{P}$ value $0.23)$. 
Table 1: Pre-operative parameters in relation to SIRS (N=97)

\begin{tabular}{|c|c|c|c|c|}
\hline \multicolumn{2}{|l|}{ Variable } & SIRS Present & SIRS Absent & $P$ value \\
\hline \multicolumn{2}{|c|}{ Stone burden $\left(\mathrm{mm}^{2}\right)($ Mean $\pm \mathrm{SD})$} & $214.32 \pm 147.19$ & $128.57 \pm 78.40$ & $0.0001(\mathrm{df}-95, \mathrm{t}=-3.725)$ \\
\hline \multirow[t]{2}{*}{ Type of stone } & $\begin{array}{l}\text { Staghorn } \\
\text { No Staghorn }\end{array}$ & 8 & 0 & 0.0001 \\
\hline & & 20 & 69 & \\
\hline \multirow[t]{2}{*}{ Hydronephrosis } & $\begin{array}{l}\text { Present } \\
\text { Absent }\end{array}$ & 36 & 21 & $\mathbf{0 . 0 4}(\mathrm{df}=-1, \chi 2=4.283)$ \\
\hline & & 33 & 7 & \\
\hline \multirow[t]{2}{*}{ Urinalysis } & $\begin{array}{l}\text { Abnormal } \\
\text { Normal }\end{array}$ & 28 & 0 & $0.0001(\mathrm{df}=1, \chi 2=41.875)$ \\
\hline & & 19 & 50 & \\
\hline \multirow[t]{2}{*}{ Urine Culture } & Positive & 12 & 18 & $0.105(\mathrm{df}=-1, \chi 2=2.622)$ \\
\hline & Negative & 16 & 51 & \\
\hline
\end{tabular}

Out of 97 patients, $28.9 \%$ developed post operative SIRS. Among patients with and without SIRS, mean stone burden was $214.32 \pm 147.19 / \mathrm{mm}^{2}$ and $128.57 \pm 78.40 / \mathrm{mm}^{2}$ respectively which was statistically significant (P value 0.0001$)$.Among 28 patients with staghorn calculi, 8 developed SIRS which was also statistically significant. (p value 0.001 ) All of the twenty eight patients with abnormal routine urine examination developed post operative SIRS ( $p$ value 0.001). Urine culture was positive in 30 patients among which 12 patients developed SIRS. (Table 1)

Table 2: Intra-operative parameters in relation to SIRS $(\mathrm{N}=97)$

$\begin{array}{lcccc}\text { Variables } & \text { SIRS Present } & \text { SIRS Absent } & \text { P value } \\ \text { Operation time (mins) } & \text { Mean } \pm \text { SD) } & 61.79 \pm 16.40 & 49.65 \pm 14.04 & \mathbf{0 . 0 0 1}(\mathrm{df}=95, \chi 2=3.672) \\ \text { No. of Access Tract } & \begin{array}{c}\text { Multiple } \\ \text { Single }\end{array} & 7 & 9 & 0.225(\mathrm{df}=1, \chi 2=2.067) \\ \text { Tract access site } & \begin{array}{l}\text { Supracostal } \\ \text { Subcostal }\end{array} & 11 & 60 & \mathbf{0 . 0 0 1}(\mathrm{df}=1, \chi 2=14.844) \\ & \begin{array}{l}\text { Present } \\ \text { Residual stone }\end{array} & 5 & 5 & 0.818(\mathrm{df}=1, \chi 2=0.053)\end{array}$

Mean operation time were $61.79 \pm 16.40$ mins and $49.65 \pm 14.04$ mins respectively in patients with and without SIRS (p value 0.001 ). Out of 97 patients, 16 had supracostal access of which 11 developed SIRS ( $p$ value 0.001 ). Number of access tracts and residual stones showed no effect in development of SIRS post operatively.(Table 2) 
Table 3: Pre-operative parameters in relation to Sepsis $(\mathrm{N}=97)$

\begin{tabular}{|c|c|c|c|c|}
\hline \multicolumn{2}{|l|}{ Variable } & Sepsis Present & Sepsis Absent & $P$ value \\
\hline \multicolumn{2}{|c|}{ Stone burden $\left(\mathrm{mm}^{2}\right)($ Mean $\pm \mathrm{SD})$} & $304.17 \pm 127.77$ & $143.37 \pm 101.23$ & $0.0001(\mathrm{df}=95, \chi 2=3.711)$ \\
\hline \multirow[t]{2}{*}{ Type of stone } & $\begin{array}{c}\text { Staghorn } \\
\text { No Staghorn }\end{array}$ & 2 & 6 & 0.076 \\
\hline & & 4 & 85 & \\
\hline \multirow[t]{2}{*}{ Hydronephrosis } & $\begin{array}{l}\text { Present } \\
\text { Absent }\end{array}$ & 5 & 52 & 0.396 \\
\hline & & 1 & 39 & \\
\hline \multirow[t]{2}{*}{ Urinalysis } & $\begin{array}{l}\text { Abnormal } \\
\text { Normal }\end{array}$ & 6 & 41 & 0.011 \\
\hline & & 0 & 50 & \\
\hline \multirow[t]{2}{*}{ Urine Culture } & Positive & 6 & 24 & 0.001 \\
\hline & Negative & 0 & 67 & \\
\hline
\end{tabular}

Out of 97 patients, $6.2 \%$ developed post operative sepsis. Mean stone burden were $304.17 \pm 127.77 / \mathrm{mm}^{2}$ and $143.37 \pm 101.23$ / $\mathrm{mm}^{2}$ respectively in patients with and without sepsis (p value 0.0001). Among 47 patients with abnormal preoperative urine analysis, six patients developed post operative sepsis (p value 0.011) and among 30 patients with preoperative positive urine culture, six developed post operative sepsis ( $\mathrm{p}$ value 0.001). Other preoperative parameters like type of stones, hydronephrosis and gender had no effect on post operative sepsis development.(Table 3)

Table 4: Intra-operative parameters in relation to Sepsis $(\mathrm{N}=97)$

\begin{tabular}{|c|c|c|c|c|}
\hline \multicolumn{2}{|l|}{ Variables } & Sepsis Present & Sepsis Absent & $\mathbf{P}$ \\
\hline \multicolumn{2}{|c|}{ Operation time (mins) $($ Mean \pm SD) } & $71.67 \pm 26.95$ & $51.93 \pm 14.05$ & $\mathbf{0 . 0 0 2}(\mathrm{df}=95, \chi 2=3.119)$ \\
\hline \multirow[t]{2}{*}{ No. of Access Tract } & Multiple & 6 & 16 & 0.585 \\
\hline & Single & 0 & 75 & \\
\hline \multirow[t]{2}{*}{ Tract access site } & Supracostal & 0 & 16 & 0.58 \\
\hline & Subcostal & 6 & 75 & \\
\hline \multirow[t]{2}{*}{ Residual stone } & Present & 2 & 14 & 0.257 \\
\hline & Absent & 4 & 77 & \\
\hline
\end{tabular}

Mean operation time were $71.67 \pm 26.95 \mathrm{~min}$ and $51.93 \pm 14.05 \mathrm{~min}$ respectively in patients with and without sepsis, which was statistically significant ( $\mathrm{p}$ value 0.002 ). Other parameters like number of access tracts, tract access site or residual stone had no affect on development of post-operative sepsis.(Table 4) 
Table 5: Univariate analysis of factors between patients with and without post-operative SIRS

\begin{tabular}{|c|c|c|c|}
\hline Variables & & OR $(95 \%$ CI $)$ & $P$ value \\
\hline $\begin{array}{l}\text { Mean Operation } \\
(\min )\end{array}$ & Time & $1.06(1.02-1.10)$ & 0.002 \\
\hline Tract access Site & & 0.121 & 0.0001 \\
\hline $\begin{array}{l}\text { Mean stone } \\
\left(\mathrm{mm}^{2}\right)\end{array}$ & Burden & $1.01(1.00-1.01)$ & 0.001 \\
\hline Type of Stone & & 0.0001 & 0.999 \\
\hline Urinalysis & & 0.0001 & 0.997 \\
\hline
\end{tabular}

Table 6: Univariate analysis of factors between patients with and without post-operative Sepsis

\begin{tabular}{|c|c|c|}
\hline Variables & OR $(95 \%$ CI $)$ & $P$ value \\
\hline Mean Stone burden $\left(\mathrm{mm}^{2}\right)$ & $1.01(1.00-1.02)$ & 0.004 \\
\hline $\begin{array}{l}\text { Mean Operation Time } \\
(\min )\end{array}$ & $1.07(1.01-1.14)$ & 0.017 \\
\hline Urinalysis & 0.0001 & 0.997 \\
\hline Urine $\mathrm{C} / \mathrm{S}$ & 0.0001 & 0.997 \\
\hline
\end{tabular}

In univariate analysis, it was observed that the mean operation time (OR 1.061, p value- 0.002, CI:1.022-1.101) and mean stone burden (OR 1.007, CI- 1.003-1.011, p value-0.001) were statistically significant in development of post operative SIRS. This states that with one minute increase in the mean operation time, the chances of developing SIRS increases by $6.1 \%$ while it increases by $0.7 \%$ with the increase in mean stone burden by $1 \mathrm{~mm}^{2}$. Similarly, mean stone burden (OR-1.009, CI:1.003-1.015, $\mathrm{p}$ value-0.004) and mean operation time (OR-1.074, CI:1.013-1.139, P value-0.017) were statistically significant in developing post operative sepsis. When there is increase in mean stone burden by $1 \mathrm{~mm}^{2}$, chances of developing sepsis increases by 0.9 percent. Similarly, chances of sepsis increases by $7.4 \%$ when there is increase in mean operation time by 1 minute.

\section{Discussion}

Current study was carried out to predict the risk factors for post-operative septic complications after PCNL. We evaluated various preoperative and intra-operative variables and their association with post-operative SIRS and sepsis.

In this study $28.9 \%$ developed SIRS in post-operative period. Similar results were seen in other studies $1,8,10-11$ while in the study in the Department of Urology of Mayo Clinic, it was observed in only $9 \%$ of patients. ${ }^{5}$ Sepsis was seen in $6.2 \%$ of patients which was similar to other results ${ }^{4,11-12}$ while Mayo clinic study showed low sepsis rates which was as low as $0.9 \%$.5

In the present study pre-operative parameters such as stone burden, types of stone and the abnormal urinalysis were associated with post-operative SIRS while gender of the patient, state of hydronephrosis or positive urine culture had no association with post-operative SIRS. Similarly, intra-operative parameters such as mean operative time was statistically associated with post-operative SIRS whereas other parameters like number and position of access tract and residual stones were not associated with post-operative SIRS. Study from Turkey showed that the factors associated with SIRS were stone burden, operation time and irrigation rate. ${ }^{11,13}$ The study conducted at Fudan University, China concluded stone size and abnormal urinalysis to be significantly associated with SIRS. ${ }^{1}$ A Thai study found that preoperative urine culture, pelvic urine culture and stone culture were positively associated with post operative SIRS. ${ }^{8}$ However, a Dutch study showed no relation between preoperative urine culture with post operative fever or SIRS. ${ }^{14}$

In the present study pre-operative parameters which were statistically associated with post-operative sepsis were stone burden, abnormal urinalysis and pre-operative positive urine culture while other variables such as type of stone and state of hydronephrosis were not associated with post-operative sepsis. This could be due to residual fragments and infective stones. Intra-operative variables which were statistically associated with post-operative sepsis were operation time whereas other parameters like number and position of access tract and residual stones were not associated with post-operative sepsis. In a study from Istanbul, sepsis was found to be associated with stone burden, operation time, fluoroscopy duration, past ipsilateral renal surgery, recurrent urinary tract infection, multiple tract and blood transfusion. ${ }^{11}$ Another study from Iraq showed positive association of sepsis with number of stones, duration of operation time, total leukocyte count, hemoglobin level and residual stone. ${ }^{4}$ 
The Mayo Clinic study found positive association of parameters like positive stone culture, struvite stones, multiple and staghorn calculi and blood transfusion with sepsis. ${ }^{5}$ Diabetes mellitus history, higher stone burden, longer operation time, increased intra-operative irrigation rate, and infectious stone composition have been found to be risk factors for urosepsis after PCNL. ${ }^{12}$

Our study showed on univariate analysis that the mean stone burden and the operation time were associated with postoperative sepsis, while pre-operative abnormal urinalysis, positive urine culture, number or site of access tract had no relation with post-operative septic complications which could be due to adequate preoperative preparation with antibiotics.

Stone burden, operation time, recurrent urinary tract infection, multiple tract, struvite stone and blood transfusion have been found to be the predictors of SIRS. ${ }^{11}$ Significant correlation between SIRS and operative time, stone size, pyelocaliectasis, the number of tracts, blood transfusion and presence of staghorn calculi have also been observed. ${ }^{10}$

The limitations of the study could be a small sample size and single centre study and multiple surgeons were involved which confounds the outcome.

\section{Conclusion}

Stone burden, types of stone, mean operation time and mean stone burden are predictive factors for postoperative infectious complications after percutaneous nephrolithotomy.

Conflict of interest: None

Funding: None

\section{Reference}

1. Yang, T.; Liu, S.; Hu, J.; Wang, L.; Jiang, H., The Evaluation of Risk Factors for Postoperative Infectious Complications after Percutaneous Nephrolithotomy. BioMed Research International 2017, 2017, 7. https:// doi.org/10.1155/2017/4832051

2. Kreydin, E. I.; Eisner, B. H., Risk factors for sepsis after percutaneous renal stone surgery. Nat Rev Urol 2013, 10 (10), 598-605. https://doi.org/10.1038/ nrurol.2013.183 PMID: 23999583

3. Gonen, M.; Turan, H.; Ozturk, B.; Ozkardes, H., Factors affecting fever following percutaneous nephrolithotomy: a prospective clinical study. J
Endourol 2008, 22 (9), 2135-8. https://doi.org/10.1089/ end.2008.0139. PMID: 18811569

4. Rashid, A. O.; Fakhulddin, S. S., Risk factors for fever and sepsis after percutaneous nephrolithotomy. Asian J Urol 2016, 3 (2), 82-87. http://dx.doi.org/10.1016/j. ajur.2016.03.001 PMID: 29264169

5. Rivera, M.; Viers, B.; Cockerill, P.; Agarwal, D.; Mehta, R.; Krambeck, A., Pre- and Postoperative Predictors of Infection-Related Complications in Patients Undergoing Percutaneous Nephrolithotomy. J Endourol 2016, 30 (9), 982-6. https://doi.org/10.1089/ end.2016.0191 PMID: 27393153

6. Singh, A. K.; Shukla, P. K.; Khan, S. W.; Rathee, V. S.; Dwivedi, U. S.; Trivedi, S., Using the Modified Clavien Grading System to Classify Complications of Percutaneous Nephrolithotomy. Current Urology 2017, 11 (2), 79-84. https://doi.org/10.1159/000447198

7. Potretzke, A. M.; Park, A. M.; Bauman, T. M.; Larson, J. A.; Vetter, J. M.; Benway, B. M.; Desai, A. C., Is extended preoperative antibiotic prophylaxis for high-risk patients necessary before percutaneous nephrolithotomy? Investig Clin Urol 2016, 57 (6), 417423. https://doi.org/10.4111/icu.2016.57.6.417 PMID: 27847915

8. Lojanapiwat, B.; Kitirattrakarn, P., Role of preoperative and intraoperative factors in mediating infection complication following percutaneous nephrolithotomy. Urol Int 2011, 86 (4), 448-52. https:// doi.org/10.1159/000324106 PMID: 21508617

9. Frazee, B. W.; Enriquez, K.; Ng, V.; Alter, H., Abnormal Urinalysis Results Are Common, Regardless of Specimen Collection Technique, in Women Without Urinary Tract Infections. Journal of Emergency Medicine 2015, 48 (6), 706-711. PMID: 25841289

10. Chen, L.; Xu, Q. Q.; Li, J. X.; Xiong, L. L.; Wang, X. F.; Huang, X. B., Systemic inflammatory response syndrome after percutaneous nephrolithotomy: an assessment of risk factors. Int J Urol 2008, 15 (12), 10258. https://doi.org/10.1111/j.1442-2042.2008.02170.x PMID: 19120510

11. Koras, O.; Bozkurt, I. H.; Yonguc, T.; Degirmenci, T.; Arslan, B.; Gunlusoy, B.; Aydogdu, O.; Minareci, S., Risk factors for postoperative infectious complications following percutaneous nephrolithotomy: a prospective clinical study. Urolithiasis 2015, 43 (1), 55-60. https:// doi.org/10.1007/s00240-014-0730-8 PMID: 25269441 
12. Liu, Y. Q.; Lu, J.; Hao, Y. C.; Xiao, C. L.; Ma, L. L., [Predicting model based on risk factors for urosepsis after percutaneous nephrolithotomy]. Beijing Da Xue Xue Bao Yi Xue Ban 2018, 50 (3), 507-513. PMID 29930421

13. Dogan, H. S.; Şahin, A.; Çetinkaya, Y.; Akdogan, B.; Özden, E.; Kendi, S., Antibiotic Prophylaxis in Percutaneous Nephrolithotomy: Prospective Study in 81 Patients. Journal of Endourology 2002, 16 (9), 649653. https://doi.org/ 10.1089/089277902761402989 PMID: 12490017

14. Draga, R. O. P.; Kok, E. T.; Sorel, M. R.; Bosch, R. J. L. H.; Lock, T. M. T. W., Percutaneous Nephrolithotomy: Factors Associated with Fever After the First Postoperative Day and Systemic Inflammatory Response Syndrome. Journal of Endourology 2009, 23 (6), 921-927. https://doi.org/10.1089/end.2009.0041 